LBL-36487

UC-414

\title{
A High Charge State Heavy Ion Beam Source for HIF
}

\author{
S. Eylon \\ Duly Research, Inc. \\ 1912 MacArthur Street \\ Rancho Palos Verdes, California 90274 \\ E. Henestroza \\ Accelerator and Fusion Research Division \\ Lawrence Berkeley Laboratory \\ University of California \\ Berkeley, California 94720
}

April 1995

This work was supported in part by the Director, Office of Energy Research, Office of High Energy Physics, of the U.S. Department of Energy under Contract No. DE-AC03-76SF00098, and by Small Business Innovation Research (SBIR) Grant No. DE-FG03-94ER81741. 


\section{DISCLAIMER}

Portions of this document may be illegible in electronic image products. Images are produced from the best available original document. 


\title{
A High Charge State Heavy Ion Beam Source for HIF
}

\author{
S. Eylon, Duly Research Inc. ${ }^{*}$. Henestroza, Lawrence Berkeley Laboratory.
}

\begin{abstract}
A high current low emittance high charge state heavy ion beam source is being developed. This is designed to deliver HIF (heavy ion Fusion) driver accelerator scale beam. Using high-charge-state beam in a driver accelerator for HIF may increase the acceleration efficiency, leading to a reduction in the driver accelerator size and cost. The proposed source system which consists of the gas beam electron stripper followed by a high charge state beam separator, can be added to existing single charge state, low emittance, high brightness ion sources and injectors. We shall report on the source physics design using $2 \mathrm{D}$ beam envelope simulations and experimental feasibility studies' results using a neutral gas stripper and a beam separator at the exit of the LBL $2 \mathrm{MV}$ injector.
\end{abstract}

\section{Introduction}

The Heavy Ion Fusion Accelerator Research (HIFAR) program is looking into the possibility of using induction linacs as drivers in an inertial confinement fusion (ICF) power plant scenario (1). An induction linac driver is envisioned now as a multiple beam accelerator (2) which after combining, acceleration, compression and final focusing hits the target in the energy plant reactor. Each accelerator's ion beam can be produced in a source injector system. A driver scale source system will deliver a 1 Amp beam with a line charge density of 0.25 micro $\mathrm{Cb} / \mathrm{m}$. The beam transverse emittance should be kept small (about $1 \mathrm{~mm}$-mrad) to allow efficient final focusing and some growth the beams combining systems. Heavy ion driver accelerator studies $(1,2)$ anticipate that the use of ion beams with a charge state $q$ higher than 1 is desirable for lowering the accelerator length and cost. We propose a source system which consists of a neutral gas beam electron stripper followed by a high charge state beam separator. The system can be added to existing single charge state, low emittance, high brightness ion sources and injectors.

\section{Source concept description}

A schematic of the proposed source system is presented in Fig. 1. The source is placed at the exit of the matching section of the LBL $2 \mathrm{MV} 0.8 \mathrm{~A} \mathrm{~K}+$ ion beam injector. The injector output beam envelope is formed in Q1, electrostatic quadrupole, to match geometrical requirements by the beam stripper design. The beam is emerging from Q1 having a sheet (rectangular) cross sections along a 60 to $70 \mathrm{~cm}$ path. The long path allows placing of the neutral gas beam stripping system and the electrostatic beam separator between Q1 and Q2.

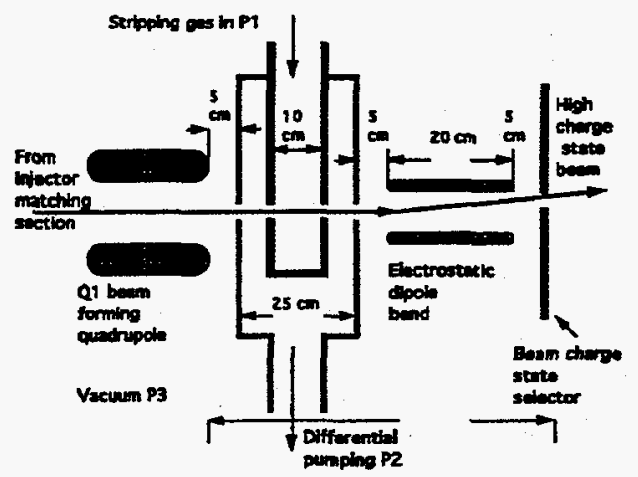

Fig. 1 High charge state source schematics

Preliminary 2D (MATCH code) beam envelope simulations to explore the physics design feasibility of the source system were performed. The simulations' results are shown in Fig. 2. One can see that the injector matching section output beam having a radius of $1.5 \mathrm{~cm}$ and $22 \mathrm{mr}$ divergence, at the beam focus (waist), is formed into a beam having a1.5 $\times 4 \mathrm{~cm}$ cross section at Q1 exit and about $1.5 \times 6 \mathrm{~cm}$ at $\mathrm{Q} 2$ input. The beam propagates along a path of $70 \mathrm{~cm}$ allowing enough space for the beam stripping and separation systems while maintaining a narrow $1.5 \mathrm{~cm}$ beam between $\mathrm{Q} 1$ and $\mathrm{Q} 2$.

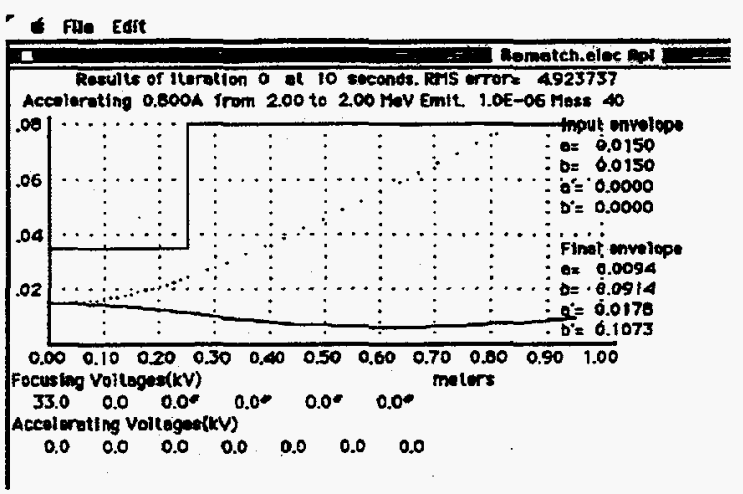

Fig. 2 Source beam envelope simulations using the 2D MATCH code. 
Separating the various high-charge-state particles from the full $(780 \mathrm{~mA})$ beam could cause emittance growth in the separated beam components. This increase in emittance could originate from non linearities due to the space-charge fields from each component as well as the fringe fields from the separator. To quantify the emittance growth after separation, we have run computer calculation using the Particle-in-Cell codes WARP3D and ARGUS. Fig. 3 shows the $\mathrm{K}^{++}$beam vertical and horizontal phase space profile at the exit of the separator. The calculation results show that the emittance of the $\mathrm{K}^{++}$sources can be maintained to about $1 \mathrm{~mm}-\mathrm{mr}$.
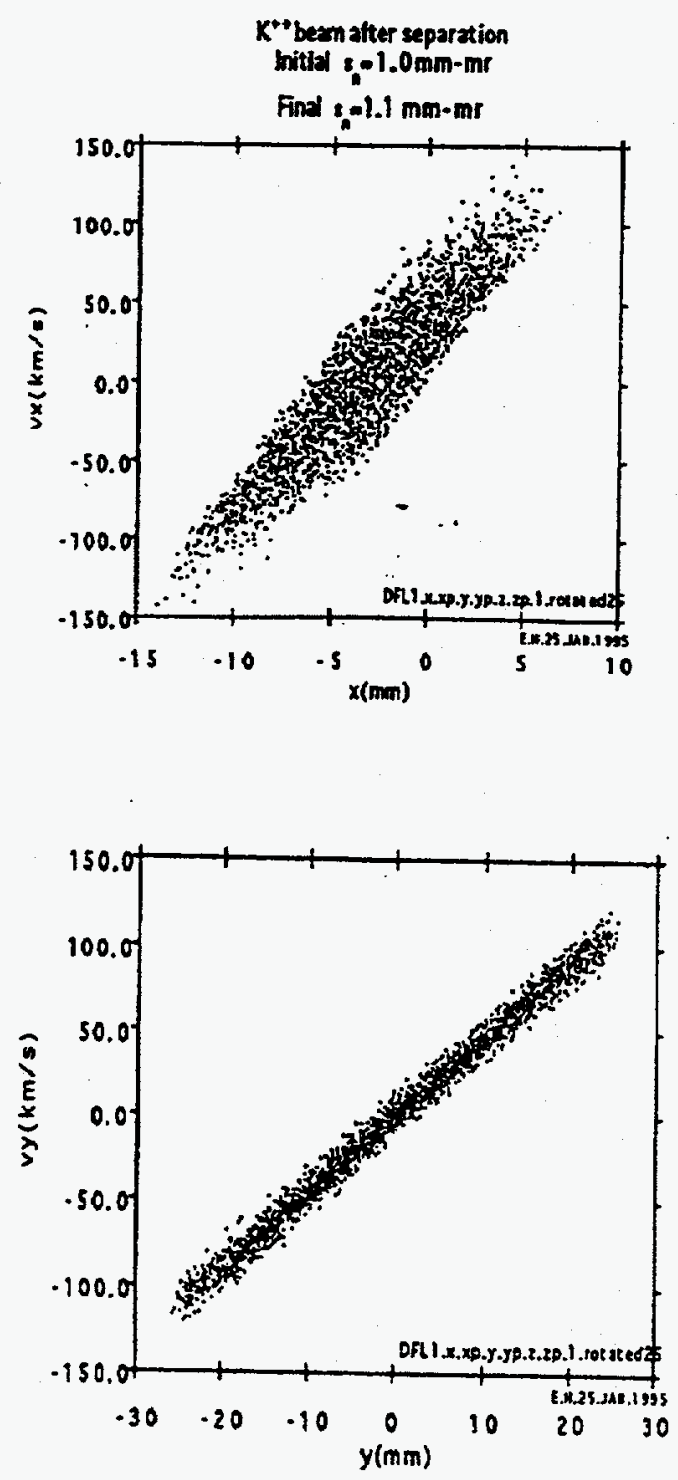

Fig. 3 Calculated $\mathrm{K}++$ beam vertical (lower) and horizontal (upper) phase at separator output. Initial normalized emittance is $1 \mathrm{~mm}-\mathrm{mr}$ and final $1.1 \mathrm{~mm}-\mathrm{mr}$.

\section{Source feasibility experiment}

The stripping gas system was placed and tested at the exit of the LBL $2 \mathrm{MV} \mathrm{K}+$ ion beam injector with in the injector existing "diagnostics box" tank. A $2 \mathrm{~mm}$ width beam aperture is used to limit the beam current, i.e., divergence, at the injector exit. The beam drifts along a distance of $12^{\prime \prime}$ within the diagnostics box into the beam stripping system. The stripping system consists of the stripping gas container (SGC) placed within the differential pumping compartment. The beam is apertured in the stripping system using four 0.5 by $20 \mathrm{~mm}$ slits at the inputs and exits of the SGC and differential pumping compartment. Using the apertures reduces the pumping system requirements and eliminates the need for high current beam containing (focusing) lenses (quadrupoles) by reducing the beam current. The beam stripping gas pressure can be controlled by a point sephire valve any were between a few mTorr up to above 1 Torr. The tilt of the SGC is fine aligned to coincide with the beam line using an adjusting system at the stripping gas inlet port. The beam out of the stripping system drifts toward the diagnostics tank exit where the following diagnostics options could be placed. Faraday cup -- to measure the beam time resolved current. Slit slit-cup -- to measure the beam current density profile , i.e., beam width (angular distribution). Measured beam width can lead to the evaluation of the beam transverse emittance assuming the slited beam (low current) is emittance dominated. Energy analyzer -- consists of a coaxial electro static beam 90 deg. bend. The analyzer is used for separating the stripped beam into the various charge states and for each charge state the measurement of the time resolved beam longitudinal energy and current.

A simple and quick way for measuring the charge state integrated stripping efficiency is by measuring the total stripped beam current using the Faraday cup at the stripping system exit. The ratio of the stripped beam current (enhanced by the higher charge) Ip taken at a stripping gas pressure $p$ to Iv at vacuum can be a measure of the charge state integrated stripping efficiency Ip/Iv or the mean charge state qm. Fig 4 presents a He stripped 1.1 MV beams pressure dependence of $\mathrm{Ip} / \mathrm{lv}$ and $\mathrm{Wp} / \mathrm{Wv}$ were $\mathrm{Wp}$ is the beam width measured at a He pressure $\mathrm{p} \mathrm{Wv}$ in vacuum. These results show that using the He gas beam stripping technique in the pressure range 50 to $100 \mathrm{mTorr}$ 
can result a high charge state beam without degrading the beam emittance.

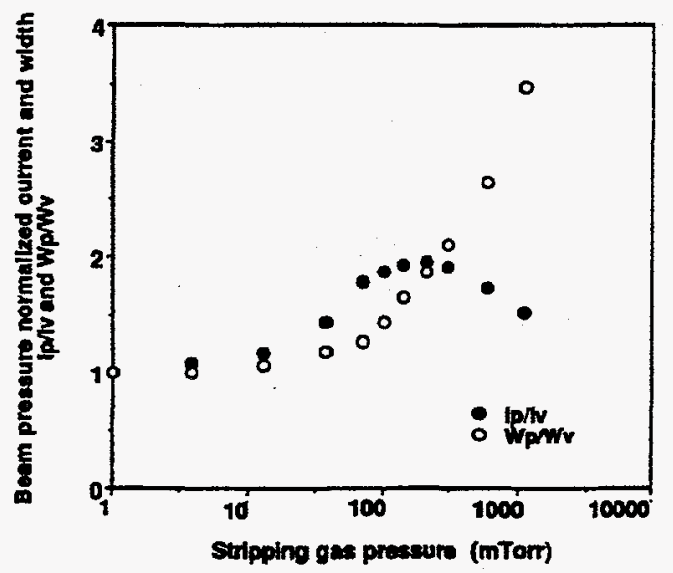

Fig. 4 Pressure normalized He stripped beam width $\mathrm{Wp} / \mathrm{Wv}$ and current Ip/Iv vs. He pressure.

Fig 5 presents the measured qm max vs beam energy $\mathrm{Ub}$ (up to $1.8 \mathrm{MV}$ ) in good agreement with calculations using Betz formula (3). The measurements were repeated using heavier neutral stripping gas like N2 and Ar showing a beginning of a growth in Wn, i.e., in the emittance in a lower stripping gas pressure.

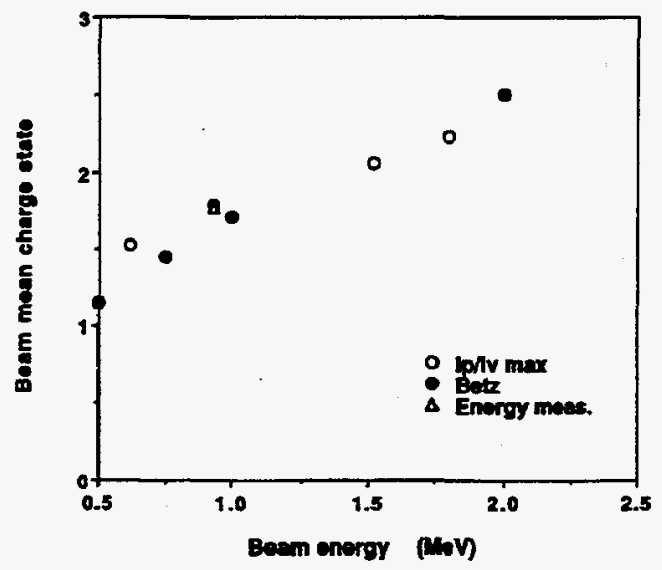

Fig. 5 Measured (Faraday cup, Energy analyzer) and calculated (Betz formula) mean charge state Qm beam energy Ub.

Beam energy and current measurements for each beam charge state were taken on a $0.9 \mathrm{MV}$ beam passing through $200 \mathrm{~m}$ Torr of He gas using the beam energy analyzer. The measured beam time resolved longitudinal mean energy profiles in the separated beams indicated (within $0.1 \%$ analyzer resolution) small or no energy losses. Integrated beam current measurements in each charge state beam led to a beam particle distribution of $47 \%, 33 \%, 15 \%, 5 \%$ and current distribution of $30 \%, 42 \%$, $19 \%, 9 \%$ in charge states $1,2,3,4$ respectfully.

\section{Discussion}

The above measurements in the $\mathrm{He}$ stripped beams were limited to beam energies of $1.8 \mathrm{MV}$. In anticipation of using the injector full energy beam ( $2 \mathrm{MV}$ ) we extrapolated the above measurements results to a $2 \mathrm{MV}$ beam using Betz formula which was shown to be in agreement with our experimental results. Thus qm of 2.3 measured in a $1.8 \mathrm{MV}$ beam can be extrapolated to $\mathrm{gm}$ of 2.5 in a $2 \mathrm{MV}$ beam. The stripped beam charge states distribution width $\mathrm{H}$ is calculated using betz (3) formula:

$$
H=0.271(Z)^{1 / 2}=1.18
$$

Where $\mathrm{Z}=19$ for a $\mathrm{K}$ ion. The increase in qm to 2.5 with an $\mathrm{H}$ of 1.18 can lead to an increase, in the charge state two beam particles content, from $33 \%$ in a $\mathrm{Ub}=0.9$ MV beam to $42 \%$ in 2 MV. Further emittance reduction is anticipated in a stripped $2 \mathrm{MV}$ beam when using emittance growth calculations (4) which accounts for beam scattering in the gas, and are consistent with our experimental for beam energies up to $1.5 \mathrm{MV}$. The above results demonstrate the feasibility of the natural gas stripping technique for achieving a source of high charge state, high current low emittance, heavy ion beam.

* Work supported in part by DOE SBIR grant no. DE-FG0394ER81741.

\section{References}

[1] D. S. Zuckerman et al. An Induction Linac Driven Heavy-Ion Fusion Systems Model. Fusion Technology Vol. 13, No. 2 Feb. 1988

[2] J. Hovingh et al. Heavy-Ion Linear Induction Accelerators as Drivers for Inertial Fusion Power Plants. Fusion Technology Vol. 13, No. 2 Feb. 1988

[3] H. D. Betz. "Heavy Ion Charge States". Applied Atomic Collision Physics, Vol. 4. Academic Press, Inc. 1983.

[4] E. P. Lee et al. General Envelope Equation for Cylindrical Symmetric Charged-Particle Beams, Appendix A. Particle Accelerators Vol. 7, 83 95. 1976. 